\title{
Ten year follow up of ulcerative colitis patients with and without low grade dysplasia
}

\author{
C H Lim, M F Dixon, A Vail, D Forman, D A F Lynch, A T R Axon
}

Gut 2003;52:1127-1132

See end of article for authors' affiliations

Correspondence to: Dr C H Lim, Department of Gastroenterology, Room 190A Clarendon Wing The General Infirmary at Leeds, Great George St, Leeds LSI 3EX, UK. ch.lim@virgin.net

Accepted for publication 25 February 2003
Background and aims: Low grade dysplasia (LGD) is believed to predispose to colorectal cancer (CRC), and proctocolectomy has been advocated when this is identified. Between 1978 and 1990, 160 patients with longstanding extensive ulcerative colitis (UC) were recruited for annual colonoscopic surveillance and 40 developed LGD at some stage. We report the outcome of this cohort 10 years after the original study ended.

Methods: Retrospective cohort study and histopathological review of the original diagnoses of LGD. The outcome of 158/160 (98.8\%) patients was established in 2000 .

Results: Of the 128 patients still alive and with an intact colon at the end of 1990, two were not traceable, 29 had LGD, and 97 had no dysplasia (controls). After 10 years, high grade dysplasia (HGD) or CRC developed in 3/29 LGD (10\%) and in 4/97 controls (4.0\%). Kaplan-Meier analysis from 1991 to death or colectomy did not show a statistically significant difference between the two groups (log rank test $p=0.63$ ). Histopathological review demonstrated the unreliability of LGD diagnosis. Agreement between pathologists was uniformly poor: kappa $<0.4$ for all comparisons.

Conclusion: LGD diagnosis is not sufficiently reliable to justify prophylactic colectomy. Conservative management of established LGD cases should not be ruled out.
$\mathrm{P}$ atients with chronic ulcerative colitis (UC) are at greater risk of colorectal cancer (CRC) than the normal population. ${ }^{12}$ This applies particularly to those with extensive colitis (proximal to the splenic flexure) and increases with the duration of disease. ${ }^{3}$ The cumulative risk of CRC is approximately $8 \% 20$ years after the initial diagnosis and rises to $18 \%$ at 30 years. ${ }^{4}$ Prophylactic colectomy 10 years after the initial attack has been suggested for UC patients ${ }^{5}$ but most clinicians and patients prefer a less radical approach. Many gastroenterology centres advocate annual colonoscopic surveillance to detect dysplasia (intraepithelial neoplasia) by histopathology as this is a precursor of CRC. ${ }^{67}$ It is generally accepted that a diagnosis of dysplasia associated lesion or mass (DALM) or high grade dysplasia (HGD) is an indication for colectomy. ${ }^{8}{ }^{9}$ If low grade dysplasia (LGD) is present, more frequent colonoscopic examinations are usually undertaken. ${ }^{10}$

It has also been suggested that better outcomes will be obtained if all patients with LGD are offered surgery ${ }^{11}$ and the recent British Society of Gastroenterology guidelines support this recommendation. ${ }^{12}$

The purpose of this study was to evaluate the long term outcome of LGD in a cohort of patients with longstanding extensive UC and who subsequently received conservative treatment. ${ }^{13} \mathrm{~A}$ second objective of the study was to assess the validity of a diagnosis of dysplasia. Previous studies have shown a low interobserver agreement for this diagnosis. ${ }^{14} 15$

\section{METHODS}

\section{Materials and patients}

In 1978, a policy of annual colonoscopic surveillance for patients with longstanding extensive UC was introduced. One to two biopsies were taken at $10 \mathrm{~cm}$ intervals along the entire colon and rectum, yielding 10-18 biopsies. When colectomy was performed, specimens were examined according to a standard protocol in which full thickness blocks are taken from areas of macroscopic abnormality, supplemented by full thickness blocks at $10 \mathrm{~cm}$ intervals from unremarkable mucosa. As a result of our experience, ${ }^{13}$ the formal policy of regular colonoscopic surveillance was stepped down in 1990. Surveillance colonoscopy was offered every three years or after an episode of relapse of UC had been treated.

In all, 160 patients participated in the original surveillance study. All had established chronic UC for longer than eight years extending beyond the splenic flexure. Forty patients were reported to have had LGD at some stage during the surveillance period up until 1990.

We have attempted to trace all patients who were under our care in 1990. Patients no longer under our follow up were sought through the local health authority. If deceased, a cause of death was obtained from death certificates. Patients no longer attending follow up were sent questionnaires asking about their well being, whether they had undergone surgery for their colitis, the date of their last colonoscopy, and other questions relevant to their outcome. We also gave these patients the option to return to our colitis clinic if they were not being followed up elsewhere. For those patients who did not respond, we contacted their general practitioner to assess their outcome. The study was approved by the local research ethics committee.

The primary outcome in this study was survival with an intact colon. We calculated the time from 1 January 1991 until the first event (HGD/CRC, colectomy, or death) encountered by each patient. We used the log rank test to compare this outcome between the LGD and no dysplasia groups.

\section{Pathology review}

All original histology slides diagnosed as dysplasia by a single specialist pathologist (MFD) were retrieved from our pathology archive. In total, we retrieved 74 LGD and one HGD slides. We also randomly chose 50 control slides from the 120 patients who were free from dysplasia during the study

Abbreviations: CRC, colorectal cancer; DALM dysplasia associated mass or lesion; HGD, high grade dysplasia; LGD, low grade dysplasia; UC, ulcerative colitis. 
160 entered surveillance programme from 1978-1990 13

30 LGD, no HGD/CRC, no colectomy, alive 7 LGD, no HGD/CRC, colectomy

1 LGD, HGD/CRC, colectomy

2 LGD, no HGD/CRC, no colectomy, died

98 no LGD, no HGD/CRC, no colectomy, alive

15 no LGD, no HGD/CRC, colectomy

5 no LGD, no HGD/CRC, no colectomy, died

1 no LGD, HGD/CRC, died

1 no LGD, cancer, unknown primary, died
Figure 1 Outcome of low grade dysplasia from 1978-1990 in 2000 CRC, colorectal cancer; HGD, high grade dysplasia; LGD, low grade dysplasia.
*29 LGD

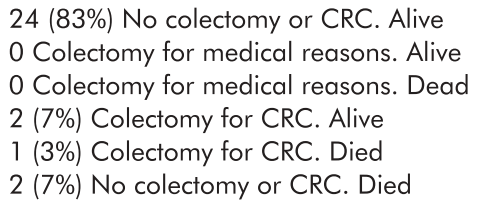

*1 non-traceable patient
*97 no dysplasio
Table 1 Methods of follow up in the low grade dysplasia (LGD) and no dysplasia groups

\begin{tabular}{lcc}
\hline Method & LGD & No dysplasia \\
\hline Personal interview & 15 & 37 \\
Patient questionnaires & 1 & 12 \\
Information from GP & 7 & 18 \\
Review of current case notes & 3 & 17 \\
Death certificate & 3 & 13 \\
& & \\
Total traceable & 29 & 97 \\
Total non-traceable & 1 & 1 \\
Total & 30 & 98 \\
\hline
\end{tabular}

period. All slides were de-coverslipped and re-stained with haematoxylin and eosin before being randomly renumbered. Five specialist gastrointestinal pathologists from different centres not involved in the original diagnosis of dysplasia were asked to assess the slides independently to score as negative for dysplasia, LGD, or HGD. There was no prior discussion of diagnostic standards or circulation of "typical" cases as we aimed to reproduce routine diagnostic practice. In order to test interobserver agreement, the diagnoses given to one LGD slide from each of the 40 patients selected at random together with the 50 non-dysplasia slides were compared using kappa statistics. This measures the degree of agreement over and above that expected by chance. ${ }^{16}$ Kappa values range from -1 to +1 , and values $>0$ indicate that agreement is greater than expected by chance. It is generally accepted that a kappa value $<0.4$ is considered poor agreement and $>0.6$ is good. We also compared the original diagnoses of LGD with the majority decision of the five pathologists (at least 3/5 pathologists).

\section{RESULTS}

A total of 160 patients entered the original prospective surveillance programme. At the end of routine surveillance in 1990, 40 had been diagnosed as having LGD on at least one occasion (fig 1). One of these 40 patients had the first diagnosis of LGD in 1979. He went on to develop HGD in 1984. He underwent colectomy and was found to have Dukes' A carcinoma. He remains alive and well today. Seven other patients had colectomies before 1990 but all were for medical reasons. One of these seven colectomy specimens contained LGD; the rest were free of dysplasia. Three patients died before 1990 including one who had undergone colectomy 10 years earlier. Their causes of death were: bronchopneumonia, ovarian cancer, and congestive cardiac failure. Therefore, 30 patients with at least one diagnosis of LGD during the surveillance period remained with an intact colon at the end of 1990 (fig l).

One hundred and twenty patients recruited to the surveillance programme had not developed dysplasia by the end of 1990. However, 15 patients had colectomies before 1990, all for medical reasons. All of these colectomy specimens were free of dysplasia. One of these 15 patients died three years after surgery from haemorrhagic shock. Seven other patients died before 1990. Three of these deaths were related to cancer. They included one disseminated colon carcinoma, one disseminated gall bladder carcinoma, and one carcinomatosis of unknown primary. There were 98 patients with an intact colon without dysplasia at the end of 1990 (fig 1).

Follow up in 2000 was available in all but two of the 128 patients (one from each group). Thus the 10 year outcome of 29 patients with LGD and 97 without dysplasia were available for analysis (table 1). The two non-traceable patients were confirmed as not registered in the NHS colorectal cancer registry. All of these patients had previously had a diagnosis of

Table 2 Characteristics of the low grade dysplasia (LGD) and no dysplasia groups

\begin{tabular}{lll}
\hline & LGD $(n=29)$ & No dysplasia $(n=97)$ \\
\hline Sex (M:F) & $13: 16$ & $48: 49$ \\
Age at the end of $1990(y)$ & $52(22-73)$ & $49(22-72)$ \\
Duration of UC since diagnosis at end of $1990(y)$ & $24(14-47)$ & $18(8-51)$ \\
No of colonoscopies at end of 1990 & $7.6(2-12)$ & $3.9(1-12)$ \\
\hline Values are mean (range). & & \\
UC, ulcerative colitis. & & \\
\end{tabular}



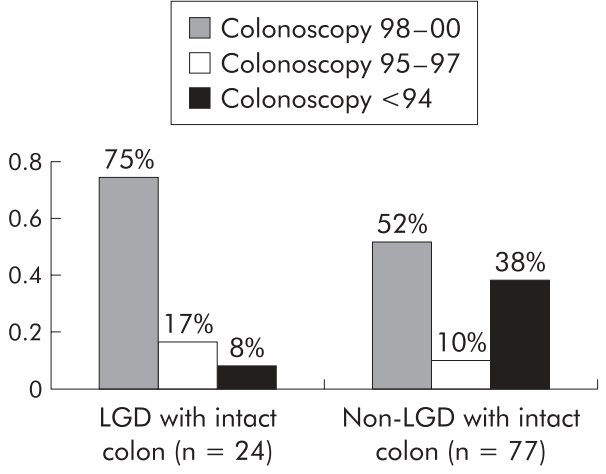

Figure 2 Year of last colonoscopy for ulcerative colitis patients with an intact colon. LGD, low grade dysplasia.

extensive colitis established at least 18 years before, and demographic data, which were comparable, are shown in table 2. Median duration of UC since diagnosis to exit date (death, colectomy, or 2000) for 126 UC patients was 26 years (range 10-55). The median follow up period after diagnosis of LGD was 17 years (range $7-22$ ).

Figure 1 shows the outcome in 2000 for all traced patients. Twenty four of 29 patients with LGD were alive with their colon intact. HGD or cancer (fig 1, table 3 ) was diagnosed in three patients (A, B, C) and all had undergone colectomy. One patient $(C)$ has since died from carcinomatosis. Two other patients from this group have died for reasons unrelated to colorectal cancer: one with ischaemic heart disease and one with renal failure secondary to methicillin resistant Staphylococcus aureus. Thus one death from CRC occurred in this group while 24 remain alive with an intact colon and no known HGD or cancer. Seventy five per cent (18/24) of these patients with an intact colon had undergone colonoscopy in the last two years (fig 2).

Of the 97 patients with no dysplasia detected during the surveillance period up to the end of 1990 (fig 1, table 3), 77 patients are alive with their colon intact. HGD or cancer was diagnosed in four patients (D, E, F, G) and all had colectomy. One patient $(\mathrm{F})$ has since died from carcinomatosis. Five other patients had colectomies for medical reasons and their colectomy specimens were free of dysplasia. One patient died three years after colectomy from pulmonary fibrosis. Thirteen patients died, including two who had undergone colectomy. Three of the 13 deaths were related to pancreatic cancer, carcinomatosis from prostatic cancer, and cholangiocarcinoma. The

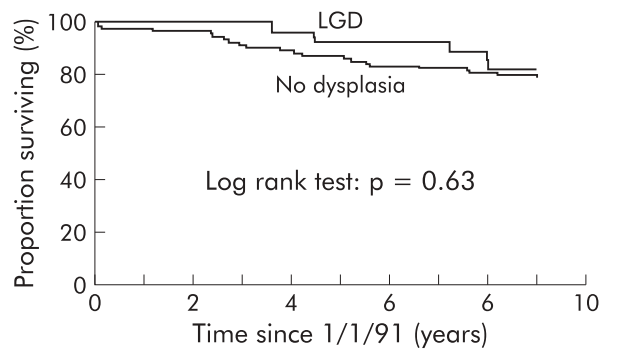

Figure 3 Comparison of time to colectomy or death between the low grade dysplasia (LGD) and control (no dysplasia) groups.

remainder died from non-cancer causes: stroke, ruptured abdominal aortic aneurysm, myocardial infarction, asthma, coronary artery disease, ischaemic heart disease, congestive cardiac failure, and "old age". There was one death from CRC and 77 have an intact colon with no evidence of cancer. More than half $(52 \%)$ of these patients have had colonoscopy in the last two years.

Survival analysis between the LGD and control (no dysplasia) groups is shown in fig 3 . Fewer LGD patients progressed to colectomy or death, but there was no statistically significant difference between the groups (relative hazard 0.78 (95\% confidence interval $0.29-2.10) ; \mathrm{p}=0.63)$.

\section{Pathology review}

The second aim of the study was to assess the validity of a diagnosis of LGD in the 40 patients. A diagnosis of LGD was made by at least one pathologist in $32 / 40(80 \%)$ dysplasia patients while two pathologists agreed with the original diagnosis of LGD in $25 / 40$ cases $(62.5 \%)$. However, a consensus diagnosis-that is, at least $3 / 5$ pathologists agreeing-was reached in only $15(38 \%)$ cases. Twelve of these 15 patients were alive with an intact colon at the end of 1990: one underwent colectomy for HGD (Dukes' A) in 1984, one had colectomy for medical reasons in 1985 and the specimen was free of dysplasia, and one died from bronchopneumonia in 1981. Of the 12 remaining patients (table 4), one (patient A) had colectomy for DALM which showed Dukes' A cancer in the transverse colon. The remaining 11 patients were alive and well in 2000. Of the 25 patients downgraded to a consensus diagnosis of no dysplasia, two (patients B, C) developed cancer during the follow up period.

For the controls, at least one pathologist diagnosed LGD in $16 / 50(32 \%)$ and a consensus diagnosis of LGD was reached in three of 50 randomly selected subjects. All three patients were

Table 3 Colorectal cancer or high grade dysplasia (HGD) arising in the low grade dysplasia (LGD) group (A-C) and no dysplasia group (D-G) between 1991 and 2000

\begin{tabular}{|c|c|c|c|c|c|}
\hline $\begin{array}{l}\text { Year of cancer } \\
\text { diagnosis }\end{array}$ & Age (y) & Sex & Duration of UC (y) & Presentation & Dukes' stage \\
\hline A: 1998 & 69 & $\mathrm{~F}$ & 44 & $\begin{array}{l}\text { LGD in 1980, 1981, 1982. Assessment colonoscopy for relapse in } \\
\text { 1998. DALM in transverse. Previous colonoscopy in 1992. Other } \\
\text { medical comorbidities prevented more earlier colonoscopy. }\end{array}$ & $A$ \\
\hline B: 1994 & 71 & M & 21 & $\begin{array}{l}\text { LGD in 1978. Surveillance colonoscopy in 1994, rectal tumour. } \\
\text { Previous colonoscopy in 1992, no dysplasia. }\end{array}$ & $\mathrm{C}$ \\
\hline C: 1995 & 43 & M & 14 & $\begin{array}{l}\text { LGD in 1989. Surveillance colonoscopy in } 1995, \text { HGD in } \\
\text { transverse. Died in } 1996 \text { from carcinomatosis. Previous colonoscopy } \\
\text { in 1993, no dysplasia. }\end{array}$ & C \\
\hline D: 1997 & 67 & M & 23 & $\begin{array}{l}\text { Surveillance colonoscopy in 1997. HGD in sigmoid. Previous } \\
\text { colonoscopy in 1995, no dysplasia. }\end{array}$ & C \\
\hline E: 1993 & 35 & M & 26 & $\begin{array}{l}\text { Defaulted from clinic and presented with relapse. Assessment } \\
\text { colonoscopy for relapse in } 1993 .\end{array}$ & $\mathrm{C}$ \\
\hline F: 1996 & 40 & M & 21 & $\begin{array}{l}\text { Defaulted from clinic and admitted to another hospital with bowel } \\
\text { obstruction in } 1996 \text {. Died in } 1998 \text { from carcinomatosis. }\end{array}$ & B \\
\hline G: 1999 & 49 & $F$ & 28 & $\begin{array}{l}\text { Surveillance colonoscopy in 1999. HGD in rectum in December } \\
\text { 1999. Surgery } 2000 \text {. Previous colonoscopy in } 1998 \text { showed active } \\
\text { colitis which was treated. }\end{array}$ & HGD only \\
\hline
\end{tabular}

DALM, dysplasia associated mass or lesion; UC, ulcerative colitis. 
Table 4 Outcome of low grade dysplasia (LGD) group before and after histopathology reassessment

\begin{tabular}{lccc}
\hline Outcome & LGD before & No LGD after & LGD after \\
\hline No colectomy or HGD/CRC. Alive & $24(83 \%)$ & $13(76 \%)$ & $11(92 \%)$ \\
Colectomy for HGD/CRC. Alive & $2(7 \%)$ & $1(6 \%)$ & $1(8 \%)$ \\
Colectomy for HGD/CRC. Dead & $1(3 \%)$ & $1(6 \%)$ & 0 \\
No colectomy or HGD/CRC. Dead & $2(7 \%)$ & $2(12 \%)$ & 0 \\
Total & 29 & 17 & 12 \\
\hline
\end{tabular}

HGD, high grade dysplasia; CRC, colorectal cancer.

alive and well in 2000. One had colectomy for medical reasons in 1980 and one had a diagnosis of LGD made in 1991 (that is, outside the study period) and still has an intact colon.

The mean frequency for the diagnosis of LGD in the 90 cases reviewed by the five pathologists was $22.2 \%$ (18-31\%) compared with $44 \%$ (40/90) for the original diagnoses. However, LGD was diagnosed by at least one pathologist in $48 / 90(53.3 \%)$ cases. With regard to interobserver agreement, the kappa coefficient between each pair of the five pathologists ranged from 0.06 to 0.39 . The kappa coefficient between the five pathologists was 0.26 . The kappa coefficient between each of the five pathologists and the original diagnosis ranged from 0.07 to 0.33 , while between the original diagnosis and majority decision was 0.17 . Therefore, agreement between these specialist gastrointestinal pathologists for LGD was uniformly poor.

\section{DISCUSSION}

The evidence cited in favour of surgery for LGD is based on 10 prospective studies. ${ }^{8} 10^{13} 17-23$ In Bernstein's analysis, between $16 \%$ and $29 \%$ of patients with untreated LGD developed a DALM, HGD, or cancer. ${ }^{11}$ In those whose initial result was negative, only $2.4 \%$ progressed to HGD. The authors concluded that immediate colectomy was indicated for all patients with HGD or LGD. However, if the data are reanalysed and patients with DALM and HGD excluded on the grounds that these are accepted indications for surgery, a different conclusion may be drawn. A total of 210 patients developed LGD during surveillance. Of these, 17 developed cancer (8\%) compared with 9/95 patients $(9 \%)$ who were "indefinite for dysplasia". These values must be compared with an $8 \%$ cumulative cancer risk at 20 years. ${ }^{4}$

The cumulative incidence of developing cancer in our series in Leeds was 9/160 (6\%), which is at the lower end of the risk estimates in the recent meta-analysis. ${ }^{4}$ Other factors however must be considered in the context of a surveillance programme. There is volunteer and selection bias on a chosen cohort of UC patients. Seven other UC patients attending our clinic developed cancer during the surveillance period (197890). ${ }^{13}$ These seven patients either defaulted from clinic before the surveillance programme was introduced or did not fulfil the criteria for surveillance. Eight other UC patients developed cancer between 1991 and 2000 (data not published). These patients were not part of the cohort of UC patients that entered the surveillance programme because they did not fulfil the criteria for surveillance prior to 1990 or they presented with CRC and UC at index colonoscopy.

More recent studies have suggested that LGD carries a high risk of progression to cancer. ${ }^{24} 25$ Lindberg et al, for example, claimed that LGD gave a predictive value of $35 \%$ for progression to HGD or cancer $(13 / 37)^{25}$ but if these data are reanalysed excluding the eight DALM patients from the LGD group, a much lower predictive value is reached. Connell et al estimated that the five year cumulative probability of LGD progressing to HGD or cancer was $54 \%{ }^{24}$ This conclusion was based on a blinded retrospective slide review by two pathologists on 84 patients who had LGD or HGD reported at least once during their surveillance colonoscopy. The five year cumulative probability of LGD progressing to HGD for the original diagnosis was $16 \%$. After histological review, only nine patients were considered to have LGD but these included two patients in whom the original diagnosis was HGD. Two cancers and two HGD were found in subsequent colectomy specimens. A recent paper reported that the five year cumulative incidence of untreated LGD progressing to advanced lesions (DALM, HGD, or cancer) was 33\% ${ }^{26}$ Median follow up in this study was 32 months. This suggests that patients who progress from LGD may do so rapidly. However, only one of the nine "advanced" lesions was cancer ${ }^{26}$; the other instances of progression were based on a subjective interpretation. Patients who do not progress quickly following LGD diagnosis may be at no measurably increased risk of progression to HGD, cancer, or death.

Every paper on screening in UC has stressed the importance of dysplastic changes in the colonic mucosa but it has been difficult to evaluate the natural history of this condition. However, reactive epithelial changes in response to inflammation may mimic LGD, making for diagnostic difficulty. Thus it is not surprising that we found considerable inter-pathologist variation. Figures 4 and 5 (both with original diagnoses of LGD) demonstrate this difficulty; one had a majority diagnosis of LGD (5/5) and the other did not (2/5). While LGD was diagnosed by at least one pathologist in $80 \%$ of the original dysplasia cases, a majority diagnosis was only achieved in $38 \%$. However, three of the original 50 "control" slides also received a majority diagnosis of LGD. When the new majority based diagnosis was assessed for survival and development of cancer, the results were little different from those based on the original diagnoses. There was no difference in survival, and the development of cancer in the consensus LGD group was $8 \%$ versus $5 \%$ for patients without dysplasia. This secondary analysis therefore confirms that LGD is of little value in predicting survival or progress to cancer. The likelihood that more of the originally non-dysplastic cases would have been re- allocated to LGD had they been subjected to review can only be surmised as proof would require reassessment of all 5695 biopsies taken during the surveillance period..$^{13}$

These diagnostic difficulties have been recognised in more recent classifications of gastrointestinal intraepithelial neoplasia in which dysplasia is categorised as normal, indefinite, low grade, or high grade. ${ }^{27}$ We did not include an "indefinite" category in our review in order to reproduce the routine diagnostic practice that prevailed during the surveillance period 1978-90. Our study confirms that the diagnosis of LGD is poorly reproduced. The low kappa values obtained with our five observers (all specialised gastrointestinal pathologists) is in keeping with other recent experience. ${ }^{14}$ Previous studies with better kappa values have been biased by employing observers from the same department (presumably similar diagnostic standards) and by inclusion of HGD cases on which agreement is uniformly better. ${ }^{24} 25$

At least two studies have suggested that LGD will develop in all UC patients if they are followed for a sufficient length of time. ${ }^{13}$ These data have been used in a decision analysis ${ }^{28}$ 


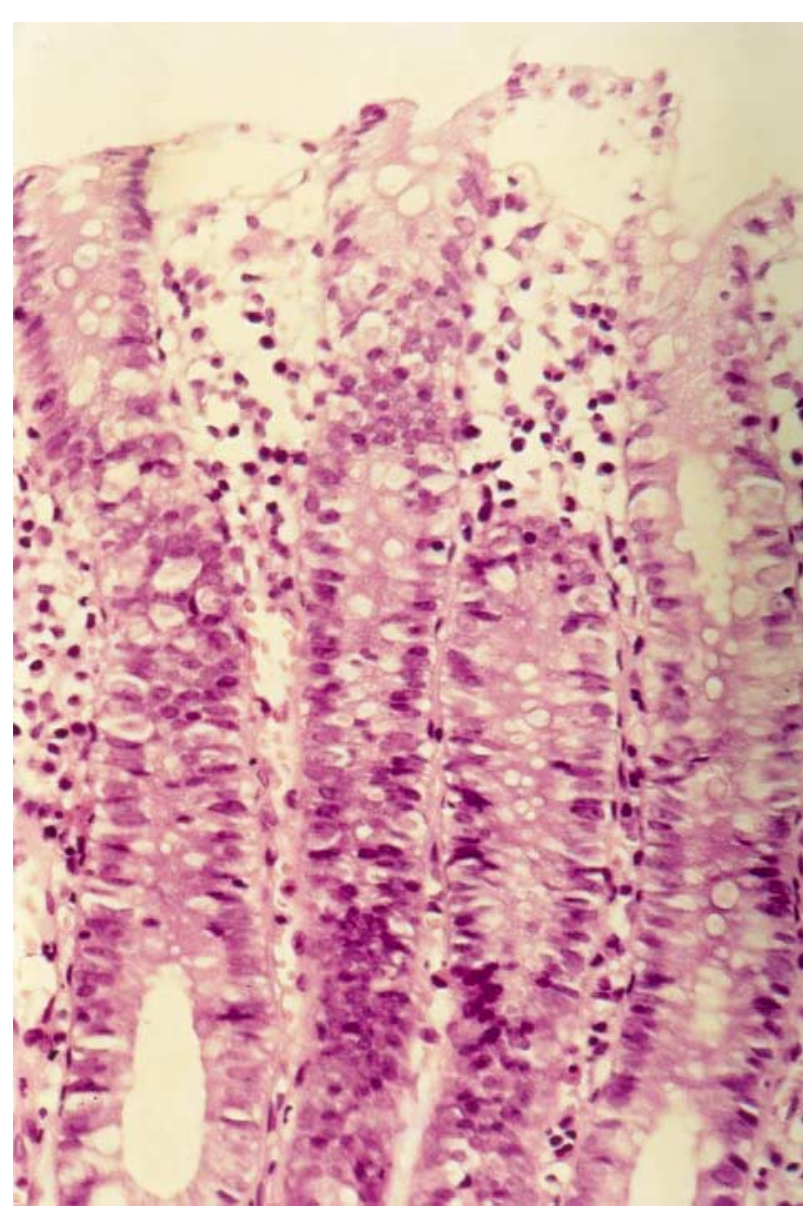

Figure 4 Low grade dysplasia by majority diagnosis.

which indicated that if 10000 patients began follow up at 10 years with a policy of colectomy at first diagnosis of LGD, 9810 would eventually undergo surgery. More frequent surveillance colonoscopy may result in greater numbers of patients with a diagnosis of LGD through false positive errors. The LGD group had on average nearly twice the number of surveillance colonoscopies by the end of 1990 (table 2). All highly variable histological diagnoses will be subject to errors which can be minimised, but not eliminated, by experience. Given the clinical context, it is likely that most pathologists will err towards dysplasia but the magnitude of the net false positive rate is difficult to estimate. If this is (say) $1-5 \%$, the implications for an individual colonoscopy are limited, but if such errors are compounded over 10 years of annual surveillance there is a substantial chance that the patient will be wrongly diagnosed as LGD. This could result in inappropriate colectomy together with an increase in workload and patient anxiety.

As with all non-experimental studies, it is important to consider the effects of possible confounding factors and biases. Perhaps the most important bias in screening studies occurs when patients with positive results are treated differently. As discussed above, more intensive monitoring is liable to lead to higher diagnosis rates. Starting the clock for this study in January 1991, when conservative management replaced routine colonoscopic surveillance, prevents such bias. However, it also means that included patients were not at similar stages of progression, with LGD cases having on average a longer duration since diagnosis. Despite this, we found no evidence that a diagnosis of LGD impairs survival with an intact colon. A greater proportion in the LGD group (10\%) progressed to cancer compared with the non-LGD group (4\%) but this was more than offset by the greater numbers of colectomies for medical reasons $(5 \% v 0 \%)$ and deaths prior to

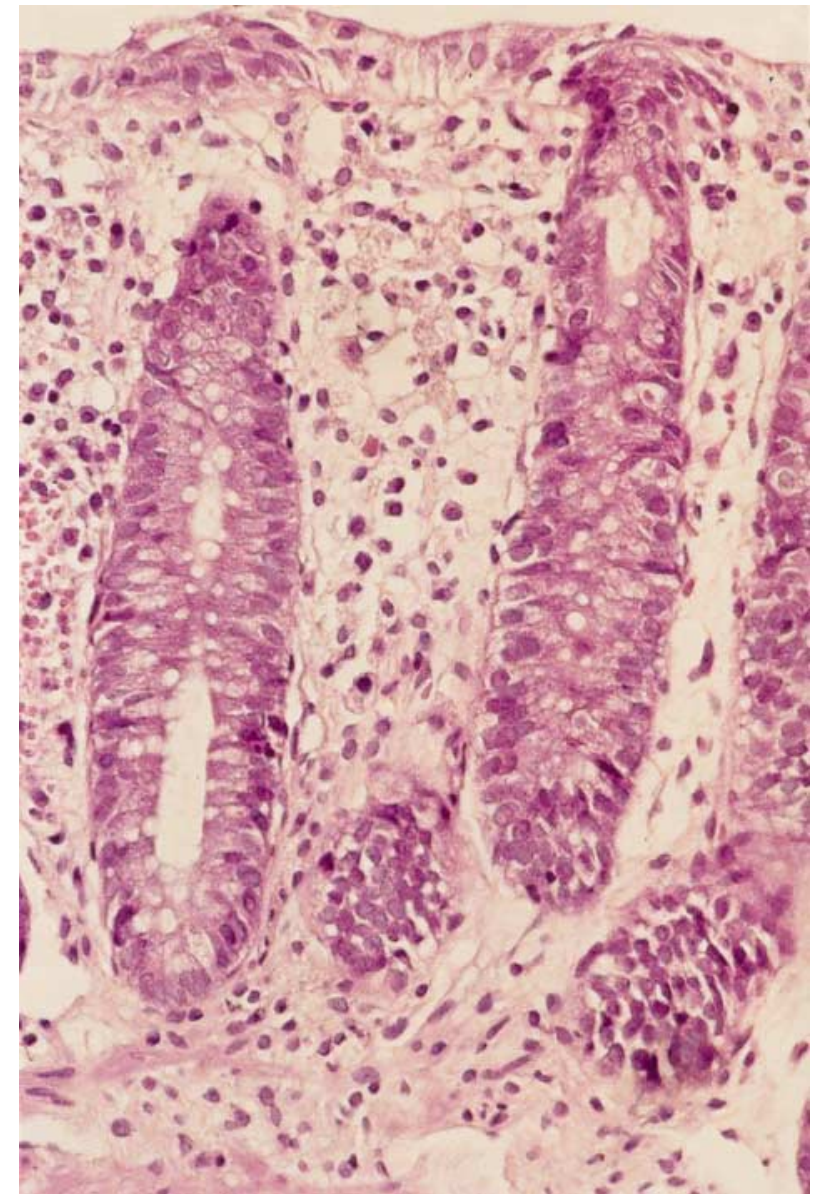

Figure 5 No dysplasia by majority diagnosis.

HGD/CRC or colectomy ( $11 \% v 7 \%$ ) in the non-LGD group. Reanalysis of our data for progression to HGD/CRC, treating medical colectomy and death as loss to follow up, also failed to show a significantly increased risk for the LGD group: relative hazard (95\% confidence interval) $2.4(0.53-10.5) ; \mathrm{p}=0.26$. There are several explanations for this study not finding evidence of differential outcome. The clearest of these is that LGD diagnosis was, and continues to be, unreliable. Secondly, our study was limited by the number of patients who entered the conservative management programme. Our patients with an established diagnosis of LGD had slightly better survival experience but the confidence interval for the hazard ratio was wide. To generalise our results, they are compatible with LGD diagnosis conferring benefit of up to $70 \%$ risk reduction, or with LGD diagnosis doubling the risk of death or colectomy.

It has been calculated that 11 biopsies are needed to detect dysplasia or cancer with $90 \%$ sensitivity in patients with underlying cancer. ${ }^{29}$ Our previous biopsy practice should have been sufficient to detect dysplasia or cancer in all of our cancer patients. One to two biopsies were taken at $10 \mathrm{~cm}$ intervals along the entire colon and rectum, yielding 10-18 biopsies per colonoscopy. Given that these lesions are likely to take more than one year to make their appearance, we can assume that at least two colonoscopies yielding 20-36 biopsies will increase the diagnostic sensitivity even further.

Although differences in cancer development between the original dysplasia and control groups were not statistically different, could they be clinically important? The protagonists of prophylactic surgery for LGD may argue that it is justified to perform 29 colectomies in order to prevent three cancers (one death). Recent published results report long term morbidity from restorative proctocolectomy that include minor pouchitis (up to 50\%), severe pouchitis (19-37\%), surgery for intestinal 
obstruction $(8-16 \%)$, pelvic sepsis $(5 \%)$, and multiple reoperations or re-laparotomies $(17-20 \%)$. The median stool frequency in 24 hours is 6-7 times, with incontinence varying between $38 \%$ and $48 \%{ }^{30}{ }^{31}$ For these reasons, we do not recommend surgery for patients who are in remission with normal bowel function unless they have HGD. Others may disagree.

In summary, our results demonstrate that diagnosis of LGD is unreliable and therefore do not support the view that UC patients with LGD should be managed differently from those with no dysplasia. Our study does not directly address the question of whether conservative management is preferable to prophylactic colectomy for those with LGD. It does fail to demonstrate any survival difference for patients with an established diagnosis of LGD. Conservative management should not be ruled out unless and until a randomised controlled trial identifies survival benefits of prophylactic colectomy for those diagnosed with LGD.

\section{ACKNOWLEDGEMENTS}

We thank the following pathologists for their participation in this study: Dr Hugh Gilmour (Edinburgh), Dr Nigel Scott (St James's Hospital, Leeds), Professor Neil Shepherd (Gloucester), Dr Bryan Warren (Oxford), and Professor Geraint Williams (Cardiff). Grant support was obtained from the NHS Executive-Northern and Yorkshire Research and Development Directorate, UK.

\section{Authors' affiliations}

C H Lim, A T R Axon, Centre for Digestive Diseases, Leeds General Infirmary, Leeds, UK

M F Dixon, Academic Unit of Pathology, University of Leeds, Leeds, UK

A Vail, Biostatistics Group, University of Manchester, Manchester, UK

D Forman, Unit of Epidemiological and Health Services Research,

Medical School, University of Leeds, Leeds, UK

D A F Lynch, Department of Gastroenterology, Blackburn Royal

Infirmary, Blackburn, UK

\section{REFERENCES}

1 de Dombal FT, Watts JM, Watkinson G, et al. Local complications of ulcerative colitis: stricture, pseudopolyposis, and carcinoma of colon and rectum. BM 1966;5501:1442-7.

2 Langholz E, Munkholm P, Davidsen M, et al. Colorectal cancer risk and mortality in patients with ulcerative colitis. Gastroenterology 1992;103:1444-51.

3 Greenstein AJ, Sachar DB, Smith H, et al. Cancer in universal and left-sided ulcerative colitis: factors determining risk. Gastroenterology 1979;77:290-4.

4 Eaden JA, Abrams KR, Mayberry JF. The risk of colorectal cancer in ulcerative colitis: a meta-analysis. Gut 2001:48:526-35.

5 Devroede G, Taylor WF. On calculating cancer risk and survival of ulcerative colitis patients with the life table method. Gastroenterology 1976;71:505-9.

6 Morson BC, Pang LS. Rectal biopsy as an aid to cancer control in ulcerative colitis. Gut 1967:8:423-34.

7 Myrvold HE, Kock NG, Ahren C. Rectal biopsy and precancer in ulcerative colitis. Gut 1974;15:301-4.
8 Blackstone MO, Riddell RH, Rogers BH, et al. Dysplasia-associated lesion or mass (DALM) detected by colonoscopy in long-standing ulcerative colitis: an indication for colectomy. Gastroenterology 1981;80:366-74

9 Lennard-Jones JE, Morson BC, Ritchie JK, et al. Cancer surveillance in ulcerative colitis. Experience over 15 years. Lancet 1983;2:149-52.

10 Woolrich AJ, DaSilva MD, Korelitz BI. Surveillance in the routine management of ulcerative colitis: the predictive value of low-grade dysplasia. Gastroenterology 1992;103:431-8.

11 Bernstein CN, Shanahan F, Weinstein WM. Are we telling patients the truth about surveillance colonoscopy in ulcerative colitis? Lancet 1994;343:71-4

12 Eaden JA, Mayberry JF. Guidelines for screening and surveillance of asymptomatic colorectal cancer in patients with inflammatory bowel disease. Gut 2002;51(suppl 5):V10-12.

13 Lynch DA, Lobo AJ, Sobala GM, et al. Failure of colonoscopic surveillance in ulcerative colitis. Gut 1993;34:1075-80.

14 Eaden J, Abrams K, McKay H, et al. Inter-observer variation between general and specialist gastrointestinal pathologists when grading dysplasia in ulcerative colitis. J Pathol 2001;194:152-7.

15 Dixon MF, Brown L, Gilmour HM, et al. Observer variation in the assessment of dysplasia in ulcerative colitis. Histopathology 1988:13:385-97.

16 Svanholm H, Starklint H, Gundersen HJ, et al. Reproducibility of histomorphologic diagnoses with special reference to the kappa statistic. APMIS 1989:97:689-98

17 Brostrom O, Lofberg R, Ost A, et al. Cancer surveillance of patients with longstanding ulcerative colitis: a clinical, endoscopical, and histological study. Gut 1986;27:1408-13.

18 Lashner BA, Silverstein MD, Hanauer SB. Hazard rates for dysplasia and cancer in ulcerative colitis. Results from a surveillance program. Dig Dis Sci 1989;34:1536-41.

19 Leidenius M, Kellokumpu I, Husa A, et al. Dysplasia and carcinoma in longstanding ulcerative colitis: an endoscopic and histological surveillance programme. Gut 1991;32:1521-5.

20 Lennard-Jones JE, Melville DM, Morson BC, et al. Precancer and cancer in extensive ulcerative colitis: findings among 401 patients over 22 years. Gut 1990:31:800-6.

21 Lofberg R, Brostrom $O$, Karlen $P$, et al. Colonoscopic surveillance in long-standing total ulcerative colitis-a 15-year follow-up study. Gastroenterology 1990;99:1021-31

22 Nugent FW, Haggitt RC, Gilpin PA. Cancer surveillance in ulcerative colitis. Gastroenterology 1991;100:1241-8.

23 Rutegard J, Ahsgren L, Stenling R, et al. Ulcerative colitis. Cancer surveillance in an unselected population. Scand J Gastroenterol 1988:23:139-45.

24 Connell WR, Lennard-Jones JE, Williams CB, et al. Factors affecting the outcome of endoscopic surveillance for cancer in ulcerative colitis Gastroenterology 1994;107:934-44.

25 Lindberg B, Persson B, Veress B, et al. Twenty years' colonoscopic surveillance of patients with ulcerative colitis. Detection of dysplastic and malignant transformation. Scand J Gastroenterol 1996-31:1195-204.

26 Ullman TA, Loftus EV, Jr, Kakar S, et al. The fate of low grade dysplasia in ulcerative colitis. Am J Gastroenterol 2002;97:922-7.

27 Schlemper RJ, Riddell RH, Kato Y, et al. The Vienna classification of gastrointestinal epithelial neoplasia. Gut 2000;47:251-5.

28 Provenzale D, Kowdley KV, Arora S, et al. Prophylactic colectomy or surveillance for chronic ulcerative colitis? A decision analysis. Gastroenterology 1995:109:1 188-96. A.

29 Rubin CE, Haggitt RC, Burmer GC, et al. DNA aneuploidy in colonic biopsies predicts future development of dysplasia in ulcerative colitis. biopsies predicts future development of
Gastroenterology 1992;103:1611-20.

30 Johnson E, Carlsen E, Nazir M, et al. Morbidity and functional outcome after restorative proctocolectomy for ulcerative colitis. Eur J Surg $2001 ; 167: 40-5$

31 Tiainen J, Matikainen M. Long-term clinical outcome and anemia after restorative proctocolectomy for ulcerative colitis. Scand J Gastroenterol 2000;35:1170-3. 